\title{
From quantum dots to quantum wires: Electronic structure of semiconductor nanorods
}

\author{
J. Planelles, ${ }^{1, *}$ M. Royo, ${ }^{1}$ A. Ballester, ${ }^{1}$ and $\mathrm{M} . \mathrm{Pi}^{2}$ \\ ${ }^{1}$ Departament de Química Física i Analítica, Universitat Jaume I, P.O. Box 224, E-12080 Castelló, Spain \\ ${ }^{2}$ Departament ECM, Facultat de Física and IN2UB, Universitat de Barcelona, E-08028 Barcelona, Spain
}

(Received 28 May 2009; revised manuscript received 24 June 2009; published 28 July 2009)

\begin{abstract}
The transition bridge between zero-dimensional quantum dots and one-dimensional quantum wires is explored theoretically by means of the construction of the addition energy spectra of nanorods with different lengths. Spin density-functional theory supplemented with full configuration interaction calculations are carried out. The addition energy spectra are qualitatively related to the single particle correlation diagram. The transition from charge-density waves to spin-density waves, characterizing the Wigner crystallization in the low density limit is shown.
\end{abstract}

DOI: 10.1103/PhysRevB.80.045324 PACS number(s): 73.21.-b, 71.15.Mb, 73.22.Gk, 85.35.Be

\section{INTRODUCTION}

Nanorods (NRs) constitute the bridge between zerodimensional quantum dots (QDs) and one-dimensional quantum wires (QWs). Investigations of the transition regime from QDs to QWs are of particular interest in the case of colloidal semiconductor nanocrystrals because size and shape control enables the synthesis of NRs with precise length and diameter, ${ }^{1}$ so that by tuning the aspect ratio one can follow the transition from zero- to one-dimensional systems.

By changing the gate voltage attached to a nanocrystal, tunnel conductance and capacitance measures yield a peak every time the number of electrons in the QD increases by one. The spacing of peaks or addition spectrum, reflects differences between the ground states with different number of electrons. This shell filling is a neat manifestation of quantum-mechanical degeneracy. While for large dots containing many electrons, oscillations in the spectrum are periodic because the single-electron charging energy is determined classically by the total dot capacitance, for a dot containing few electrons both, quantum effects reflecting the symmetry of the confining potential and electron-electron interactions, lead to modifications of the oscillations so they are no longer periodic, ${ }^{2}$ thus revealing the shell structure of the dot.

Tunneling spectra of single CdSe NRs of radii and lengths ranging between 1.8-3.2 and 11-60 nm, respectively, have been reported. ${ }^{3}$ The spectra were measured with the tip at a distance avoiding charging effects. On the basis of a theoretical formalism for QWs, the observed peaks were assigned to low-lying conduction bands with azimultal angular momentum $m_{z}=0,1,2$, but no discretization of these bands originated from confinement was resolved. Similar InAs NR spectra with not well resolved conduction band states has also been published. ${ }^{4}$ Not many experimental addition spectra of short NRs can be found in the literature. Long and wide Si NWs display a uniform peak height and width and equidistant spacing in the current vs gate voltage spectrum ${ }^{5}$ while shorter thinner Si NRs show a more irregular spectra, ${ }^{6}$ thus revealing a shell structure.

Theoretical understanding of QD addition spectra usually rely on isotropic and anisotropic harmonic confining potentials. $^{2,6,7}$ Thus, two-dimensional (2D) harmonic QDs has a $2,4,6,8 \ldots$ degeneracy pattern yielding high peaks in the addition energy spectrum at $N=2,6,12,20 \ldots$ electrons, ${ }^{8}$ this structure being partially destroyed by elliptical deformation of the QD. ${ }^{9}$ On the other hand, the confining potential of a spherical colloidal nanocrystal, typically synthesized by wet chemistry methods, is better represented by a steplike hard wall potential, ${ }^{10}$ which corresponds to the band gap difference between neighboring materials. This confining potential yields a $2,6,10 \ldots$ degeneracy pattern which shows up at $N=2,8,18 \ldots$ electrons. NRs are an elongated variant of colloidal QDs, ${ }^{1}$ i.e., may be considered as a QD with a highly anisotropic confining potential, which is resposible for some remarkably strong Coulomb interactions. ${ }^{11,12}$ Then, the transition from QD to NR is not only characterized by a symmetry change of the confining potential, but additionally by a transition from strong to weak confinent regime. Monitoring this change of symmetry and confining regime in the addition spectra is the aim of this work. We carry out local spin-density-functional theory calculations supplemented with full configuration interaction (FCI) computations. An interesting $2 \mathrm{D}$ model calculation, correlating the addition spectra of squared and rectangular boxes with the same area, shows the abovementioned double transition. ${ }^{13}$ Our calculations, which correspond to a truly three-dimensional (3D) NR system of constant section and variable length, display a different addition energy pattern, as it corresponds to different confining potentials, but converges toward qualitative similar profiles in the limit of weak confining regime. In the low-density limit we also find a transition from chargedensity waves (CDW) with $N / 2$ peaks in the density profile vs the NR axis and preserved spin symmetry to spin-density waves (SDW) with $N$ peaks (Wigner crystallization) and broken spin symmetry. ${ }^{14}$ Further, the fully spin polarized state is reached. ${ }^{15}$

\section{THEORY}

Local spin density functional theory (LSDFT), which has given satisfactory results in the study of related structures, $8,13,18,19$ is employed in the present work. Numerical integration of the Kohn-Sham equations is carried out. Details of the method followed are described in Ref. 8. The 
exchange-correlation functional is taken as a sum of the Dirac exchange functional of a homogeneous electron gas and the correlation functional parametrized by Perdew and Zunger. ${ }^{20}$ The electron effective mass and the dielectric constant, screening the electron-electron interaction, are assumed to be equal to that of the NR bulk material. ${ }^{21}$ The confining potential is the same we used in a previous study of NRs, ${ }^{22}$ namely, zero within the NR composed by a cylinder of radius $R$ and variable length $L$, attached to two hemispherical caps of the same radius $R$, and $V^{\text {out }}$ elsewhere. Some FCI calculations are carried out for $N=4,6,8$ electrons and $L=10 \mathrm{~nm}$, in order to confirm the density profiles obtained with LSDFT. As we will see, in these cases only $m_{z}$ $=0$ orbitals come into play. Then, we select a basis set of orthogonalized floating spherical Gaussian functions. The original nonorthogonal spherical Gaussian functions $g(\mathbf{r})$ $=e^{-\alpha\left(\mathbf{r}-\mathbf{R}_{i}\right)^{2}}$ are defined by the exponent $\alpha$ and the position $\mathbf{R}_{i}$ where they are centered. The exponent $\alpha$ is fitted variationally in a $L=0 \mathrm{NR}$ (sphere) calculation in which a single Gaussian function is employed. The floating Gaussian functions are centered along the NR axis, equally spaced, so that they saturate the space. The number of Gaussians is increased up to convergence in energy. The same density of Gaussian functions along the NR axis is employed in all calculations. Once the primitive Gaussian functions are established, orthonormalization yielding a new basis set which most closely resemble the original basis functions, i.e., a symmetric orthogonalization employing $S^{-1 / 2}$, the inverse of the original basis set metrics square root, is carried out. ${ }^{23}$ With this orthonormal basis set, the many-body Hamiltonian reads in second quantization language,

$$
H=\sum_{p q}^{M} h_{p q} a_{p}^{+} a_{q}+\sum_{p q r s}^{M} g_{p q r s} a_{p}^{+} a_{q}^{+} a_{r} a_{s},
$$

where $a_{p}^{+}, a_{q}$ are fermion creator and annihilator operators, and $h_{p q}$ and $g_{p q r s}$ the one- and two-electron integrals. Except for the contribution of the potential energy coming from the confining potential to the single-electron integrals, which is calculated numerically, all integrals are computed analytically. ${ }^{23}$

In order to carry out FCI calculations, once the oneelectron basis set $\left\{\phi_{p}\right\}_{p=1}^{M}$ is selected, we build all possible $N$-body Slater determinants $\Phi_{\alpha}=\operatorname{det}\left\{\phi_{1} \phi_{2} \ldots \phi_{N}\right\}$ out of them. In a second step, we project Hamiltonian (1) onto the basis set of Slater determinants yielding matrix elements $\left\langle\Phi_{\alpha}|H| \Phi_{\beta}\right\rangle$. Finally, we proceed with the diagonalization.

\section{RESULTS AND DISCUSSION}

We study the electronic structure of CdSe NRs. The geometry, described in the previous section, is defined by the NR radius, fixed to $R=2 \mathrm{~nm}$, and the length, which we range from $L=0$ up to $L=55 \mathrm{~nm}$. The material parameters employed are those of the bulk material, ${ }^{24}$ namely, effective mass $m^{*}=0.13$ and dielectric constant $\varepsilon=9.2$. The height of the confining potential is set to $V^{\text {out }}=4 \mathrm{eV}$.

In Fig. 1 we show the low-lying single particle noninteracting energy $(s p)$ levels obtained within the effective mass

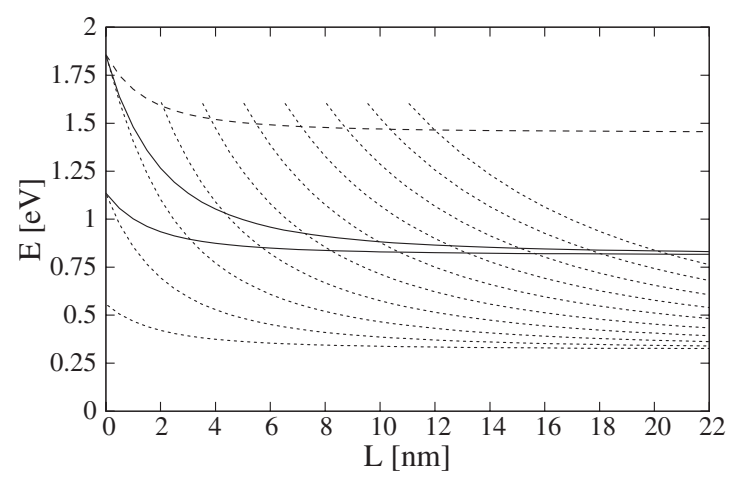

FIG. 1. Energy of the lowest NR $s p$ noninteracting energy levels (eV) vs the NR length $L(\mathrm{~nm})$. Dotted, full and dashed lines correspond to orbitals with azimutal quantum number $m_{z}=0, m_{z}= \pm 1$, and $m_{z}= \pm 2$, respectively.

approach formalism. We represent $s p$ energy vs NR length $L$. Dotted lines correspond to orbitals with $m_{z}=0$, full lines to $m_{z}= \pm 1$ and dashed lines to $m_{z}= \pm 2$. At $L=0$ we can see the typical spherical $1-3-5 \ldots$ degeneracy $(2-6-10 \ldots$ including spin). As $L \neq 0$, the symmetry reduces from spherical to axial, with just one- and two-dimensional irreducible representations, i.e., with just nondegenerate and twofold degenerate orbitals (degeneration 2 and 4 including spin). In the figure one can also see how very excited $m_{z}=0$ orbitals at $L=0$ stabilize as $L$ increases, converging toward the lowest conduction band of the infinite wire. This accumulation of $m_{z}=0$ states looks similar to the formation of Landau bands in the Fock-Darwin energy spectra vs magnetic field. Additionally, despite only two $m_{z}= \pm 1$ and one $m_{z}= \pm 2$ levels are included in Fig. 1, one can envisage the formation of the second conduction band and the bottom of the third one. These bands correspond to the ones observed in Ref. 3. Also, the figure allows us to determine degeneracy/ quasidegeneracy vs $L$, which shows up as peak patterns in the addition energy spectra. Thus, one can foresee that for $L=0$ (sphere) the consecutive electron charging up to $N$ $=20$ yields three strong peaks at 2,8 , and 18 electrons, while in the case of $L=20 \mathrm{~nm}$ a featureless addition energy spectrum will be obtained. As can be seen in Fig. 2, these qualitative trends are fully confirmed by the LSDFT calculations, where exchange and correlation are included. In this figure we represent addition energies $\Delta_{2}(N)=E(N+1)-2 E(N)$ $+E(N-1)$, with $E(N)$ being the ground state total energy of the $N$-electron NR system, vs the number $N$ of electrons for NRs of different length. From top to bottom, we represent $L=0$ up to $L=22 \mathrm{~nm}$ in steps of $\Delta L=2 \mathrm{~nm}$. Addition energies are given in $\mathrm{eV}$. However, in order to group all the results within a single figure, the different profiles have been offset $400 \mathrm{meV}$. For example, addition energies for $L=0$ should be subtracted by an amount of $4.4 \mathrm{eV}$.

As pointed out above, Fig. 2 qualitatively reproduces the degeneracy pattern observed in Fig. 1. Thus, for $L=0$, in addition to three main peaks at $N=2,8$, and 18 electrons, which correspond to the filling of the three lowest shells, one can see secondary, less intense, peaks at $N=5$ and $N=13$ electrons, corresponding to half fillings. As shown in Fig. 1, as the NR gets longer, excited $m_{z}=0$ levels fall below the 


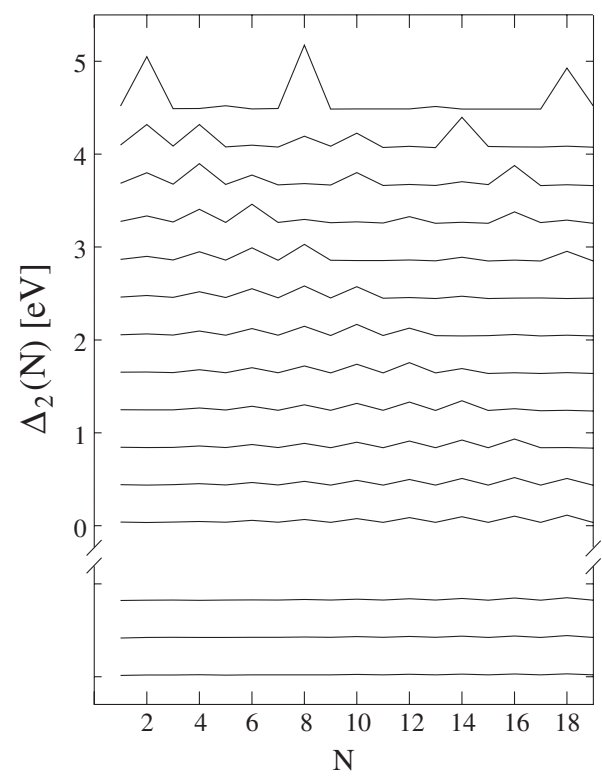

FIG. 2. Addition energy spectra $(\mathrm{eV})$ for $\mathrm{NR}$ of increasing length $L$ vs the number $N$ of electrons. Increasingly larger NRs are represented from top to bottom, starting with $L=0$ (spherical QDs) up to $L=22 \mathrm{~nm} \mathrm{NR}$, in steps $\Delta L=2 \mathrm{~nm}$. The different profiles have been offset $400 \mathrm{meV}$. The three almost flat profiles at the bottom correspond to the low-density NRs of length $L=40,45$ and $55 \mathrm{~nm}$.

low-lying $m_{z}= \pm 1$ one, and occupied orbitals become closer in energy, i.e., the density of states increases. This leads to a change in peak pattern and a general lowering of the addition energies (see Fig. 2). For long NR, e.g., $L=20 \mathrm{~nm}$, we also see in this figure that the spectrum starts almost flat at low $N$ and wrinkles as this number increases. It is related to a growth in the energy gap between neighboring levels of the $m_{z}=0$ lowest band as we move toward higher energies, i.e., as we fill the NR with a larger number of electrons (see Fig. 1). For very large $L$ we reach the Wigner crystallization limit and, as expected, an almost flat curve for all $N$ (see bottom of Fig. 2). We will turn our attention to this limit later on in the paper.

Details of the addition spectra vs $L$ can be understood with the help of Fig. 1. For example, as can be seen in this figure, if we fill with $N=18$ electrons an $L=2 \mathrm{~nm} \mathrm{NR}$, we have the following electronic configuration: $\begin{array}{lllllllll}0^{2} & 0^{2} & 1^{4} & 0^{2} & 1^{4} & 2^{4} \text {, where the exponents represent the num- }\end{array}$ ber of electrons filling the orbital whose $m_{z}$-modulus is being powered. This configuration will show main peaks at $N$ $=2,4,8,10,14,18$ electrons (filled shells) and secondary peaks at $N=6,12,16$ (half fillings). This result is confirmed in Fig. 2, although this figure also reveals that the peak at $N=18$ does not exist. Coming back to Fig. 1, one can see that at $L=2 \mathrm{~nm}$ there is a quasidegeneration of the first $m_{z}$ $= \pm 2$ and the fourth $m_{z}=0$ levels, so that instead of an electronic configuration $2^{4}$ one may think of $(2,0)^{4}$, which does not yield a main peak in the addition energy spectrum at $N$ $=18$.

As a main result of this set of calculations one may conclude that for CdSe NRs, with a shape ratio larger than 6 , the addition energy spectra will become featureless. Since CdSe, with an effective mass of 0.13 and a dielectric constant of
9.2, has an effective Bohr radius of about $3.8 \mathrm{~nm}$, one can foresee that semiconductor materials such as InAs, with effective Bohr radius about ten times larger, will display features revealing a given degeneracy pattern until much larger shape ratios.

We further explore the low-density limit. A comprehensive study on phase diagrams for interacting fermions in onedimensional periodic potentials ${ }^{25}$ brings a description of the electron system in terms of fields representing fluctuations in the spin and charge densities. The existence of localization and magnetic transitions in the low-density limit, have also been reported for infinite ${ }^{26}$ and finite ${ }^{27}$ wires defined by harmonic confinement potentials. In order to explore here the low-density limit of free standing NRs we calculate the LSDFT ground state of long but finite NRs. The obtained results are summarized in Fig. 3, where total (full thick line), spin up (dashed line), and spin down (dotted line) density profiles vs the NR axis, for NR lengths $L=40,45$, and $55 \mathrm{~nm}$, and populations $N=4,6,8$ electrons, are represented. For the sake of comparison, results for a short $L=10 \mathrm{~nm}$ NR are also enclosed. In this case, also the total density profile coming from FCI calculations $(+)$ is shown. The reason of these FCI calculations is to confirm the observed concentration of total density distribution toward the ends of the NR. A similar behavior was already noticed in the model calculation of Ref. 13, in which LSDFT calculations were carried out, and contrast with the results in Ref. 27, where the total density is more pronounced at the center, due to the harmonic confinement employed in this last study. The same concentration of total density distribution toward the ends of the NR can also be seen in 16, where CI calculations of an effective onedimensional (1D) model is performed. In this last calculation, the CI expansion employs the numerical $s p$ eigenfunctions of the single-electron Hamiltonian. Provided the recently reported difficulties concerning CI calculations based on $s p$ orbitals owing to a lack of saturation of the space along the axis of quasi-1D systems, ${ }^{12}$ we have checked the obtained results with FCI calculations, as outlined in the previous section, which employ a basis set ensuring saturation along the NR axis. Comparison in Fig. 3 confirms the observed trend in the density profile of NR given by LSDFT calculations, which is probably related to the minimization of the electron-electron repulsion.

Figure 3 shows that for $L=40 \mathrm{~nm}$ and $N=4,6$, and 8 , the so-called charge-density waves, with $N / 2$ maxima in the density profile and same spin up and down density profiles (i.e., with preserved spin symmetry) appear. At $L=45 \mathrm{~nm}$, the more diluted $N=4$ electron system shows up another phase while less diluted, $N=6$ and 8 electron systems remain in the CDW phase. This phase, referred to as spin-density wave, shows $N$ maxima in the density profile and broken spin symmetry. ${ }^{14}$ This corresponds to a Wigner-like crystallization, in which the electrons behave like classical density charge arranged within the NR to avoid each other as much as possible. This phase transition, referred to as spin-Peierls transition, is paralleled by a pronounced increasing of the Fermi gap. ${ }^{27}$ We also observe this opening. For example, for $N=6$, the ratio between the occupied bandwidth (energy difference between the lowest and highest occupied levels) and the Fermi gap (energy difference between the highest occu- 


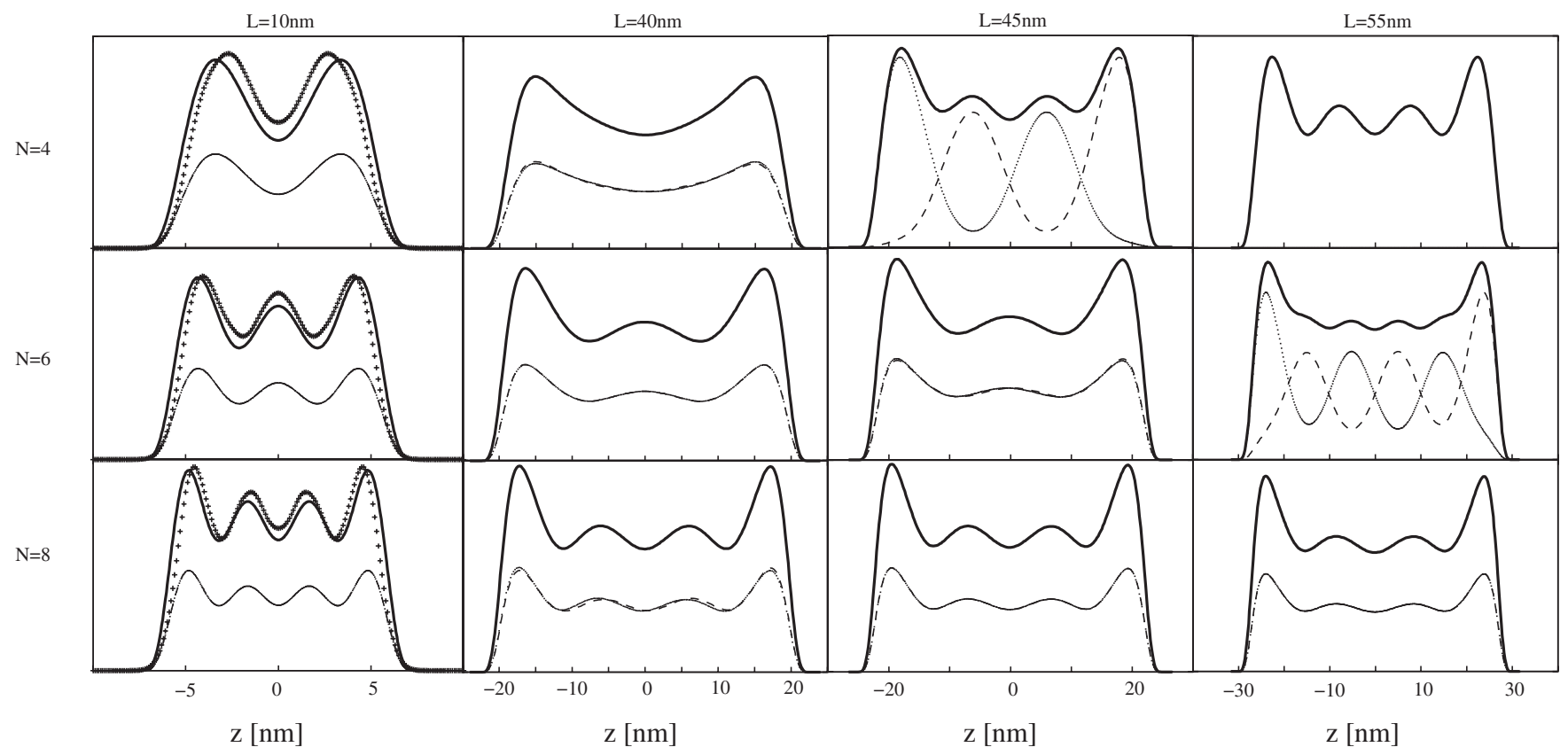

FIG. 3. Electron density profiles along the NR $z$ axis, corresponding to NRs of different lengths $L$, populated with different number $N$ of electrons. Columns from left to right cover the different lengths: $L=10,40,45$, and $55 \mathrm{~nm}$, respectively. From top to bottom rows, increasing number $N$ of electrons, $N=4,6$, and 8 . Total (full thick line), spin up (dashed line), and spin down (dotted line) density profiles are shown. For $L=10 \mathrm{~nm}$, FCI total density (+) is also enclosed. In those cases in which spin up and down density are equal, dashed and a dotted lines overlap and almost a full thin line profile results.

pied and lowest unoccupied levels) is 1.17 at $L=45 \mathrm{~nm}$ (CDW phase) and 0.51 at $L=55 \mathrm{~nm}$ (SDW phase). At $L$ $=55 \mathrm{~nm}$ the $N=8$ system still remains in the CDW one while the most diluted $N=4$ system undergoes a transition to a fully spin polarized state. ${ }^{15}$

\section{CONCLUDING REMARKS}

We have investigated the electronic structure of semiconductor NRs. Most calculations employ LSDFT. A few FCI calculations supporting LSDFT results have been also performed. We study the transition from zero-dimensional QDs, with well defined energy levels, to one-dimensional QWs, with energy bands in one dimension. We monitor this transi- tion with the addition energy spectrum which reveals changes in degeneracy and the increasing role of the electron-electron interactions as $L$ increases. The obtained addition spectra are qualitatively related to the $s p$ correlation diagram vs the NR length $L$. The limit of low-density is explored and the transition from CDW to SDW and, further, to the fully spin polarized phase is shown and discussed.

\section{ACKNOWLEDGMENTS}

We wish to thank J. I. Climente for helpful comments. Continuous support from MCINN Projects No. CTQ200803344 and No. FIS2008-00421/FIS and UJI-Bancaixa Project No. P1-1B2006-03 is gratefully acknowledged. *josep.planelles@qfa.uji.es

${ }^{1}$ X. Peng, L. Manna, W. Yang, J. Wickham, E. Scher, A. Kadavanich, and A. P. Alivisatos, Nature (London) 404, 59 (2000); S. Kan, T. Mokari, E. Rothenberg, and U. Banin, Nature Mater. 2, 155 (2003).

${ }^{2}$ S. Tarucha, D. G. Austing, T. Honda, R. J. van der Hage, and L. P. Kouwenhoven, Phys. Rev. Lett. 77, 3613 (1996).

${ }^{3}$ D. Katz, T. Wizansky, O. Millo, E. Rothenberg, T. Mokari, and Uri Banin, Phys. Rev. Lett. 89, 086801 (2002).

${ }^{4}$ D. Steiner, D. Katz, O. Millo, A. Aharoni, S. Kan, T. Mokari, and U. Banin, Nano Lett. 4, 1073 (2004).

${ }^{5}$ S. Huang, N. Fukata, M. Shimizu, T. Yamaguchi, T. Sekiguchi, and K. Ishibashi, Appl. Phys. Lett. 92, 213110 (2008).
${ }^{6}$ K. H. Cho, Y. C. Jung, B. H. Hong, S. W. Hwang, J. H. Oh, D. Ahn, S. D. Suk, K. H. Yeo, D.-W. Kim, D. Park, and W.-S. Lee, Appl. Phys. Lett. 90, 182102 (2007).

${ }^{7}$ S. M. Reimann and M. Manninen, Rev. Mod. Phys. 74, 1283 (2002).

${ }^{8}$ M. Pi, A. Emperador, M. Barranco, and F. Garcias, Phys. Rev. B 63, 115316 (2001).

${ }^{9}$ D. G. Austing, S. Sasaki, S. Tarucha, S. M. Reimann, M. Koskinen, and M. Manninen, Phys. Rev. B 60, 11514 (1999).

${ }^{10}$ M. Pi, M. Royo, and J. Planelles, J. Appl. Phys. 100, 073712 (2006); M. Royo, J. Planelles, and M. Pi, Phys. Rev. B 75, 033302 (2007); J. Planelles, M. Royo, and M. Pi, J. Appl. Phys. 102, 094304 (2007). 
${ }^{11}$ A. Shabaev and Al. L. Efros, Nano Lett. 4, 1821 (2004); J. I. Climente, M. Royo, J. L. Movilla, and J. Planelles, Phys. Rev. B 79, 161301(R) (2009).

${ }^{12}$ J. Planelles, J. I. Climente, M. Royo, and J. L. Movilla, J. Phys.: Condens. Matter 21, 215801 (2009).

${ }^{13}$ E. Räsänen, H. Saarikoski, V. N. Stavrou, A. Harju, M. J. Puska, and R. M. Nieminen, Phys. Rev. B 67, 235307 (2003).

${ }^{14}$ The spin-symmetry breaking observed in LSDFT is not observed in CI calculations (Ref. 16). An internal-space spin order shows up instead, so that this phase can be identified as the tendency of mean field to mimic such an internal ordering.

${ }^{15}$ The real existence of the fully polarized phase should be taken with caution, in the sense that LSDA generally overestimates exchange and correlation in extremely diluted systems (see, e.g., Ref. 17). In the particular $N=4, L=55 \mathrm{~nm}$ case, represented in Fig. 3, one may converge both the fully polarized and the SDW phase. The total energy of the fully polarized ground state is found to be $1.26 \mathrm{eV}$, just $0.3 \mathrm{meV}$ below the SDW state. Then, owing the abovementioned exchange and correlation overestimation of LSDA in diluted system, the obtained result may be just considered as a signature of attainment of the extremely diluted regime.

${ }^{16}$ B. Szafran, F. M. Peeters, S. Bednarek, T. Chwiej, and J. Ad- amowski, Phys. Rev. B 70, 035401 (2004).

${ }^{17}$ F. Pederiva, A. Emperador, and E. Lipparini, Phys. Rev. B 66, 165314 (2002).

${ }^{18}$ M. Pi, A. Emperador, M. Barranco, F. Garcias, K. Muraki, S. Tarucha, and D. G. Austing, Phys. Rev. Lett. 87, 066801 (2001).

${ }^{19}$ A. Wensauer, O. Steffens, M. Suhrke, and U. Rössler, Phys. Rev. B 62, 2605 (2000).

${ }^{20}$ J. P. Perdew and A. Zunger, Phys. Rev. B 23, 5048 (1981).

${ }^{21}$ Since no specific NR environment is determined, possible polarization effects coming from the dielectric mismatch between the $\mathrm{NR}$ and the surrounding medium are disregarded.

${ }^{22}$ F. Rajadell, J. I. Climente, J. Planelles, and A. Bertoni, J. Phys. Chem. C 113, 11268 (2009).

${ }^{23}$ A. Szabo and N. S. Ostlund, Modern Quantum Chemistry (McGraw-Hill, New York, 1989).

${ }^{24}$ U. E. H. Laheld and G. T. Einevoll, Phys. Rev. B 55, 5184 (1997).

${ }^{25}$ E. B. Kolomeisky and J. P. Straley, Rev. Mod. Phys. 68, 175 (1996).

${ }^{26}$ K. Kärkkäinen, M. Koskinen, S. M. Reimann, and M. Manninen, Phys. Rev. B 72, 165324 (2005).

${ }^{27}$ S. M. Reimann, M. Koskinen, and M. Manninen, Phys. Rev. B 59, 1613 (1999). 